\section{A simple outfit for the detection of bacteriuria}

\section{W. A. SUTCLIFFE AND R. GLEDHILL From the Public Health Laboratory, Sheffield}

Many methods of detecting bacteriuria have been described. Probably the ones most favoured have been the 'dip spoon' of Mackey and Sandys (1965) and the improved 'dip slide' of Naylor and Guttman (1967) which has been modified by severalcommercial firms.

The difficulties of these methods have primarily been with the provision of a sterile wide-mouthed container for the patient to collect the sample of urine before using the dip slide, and secondly the cost of the outfit provided, especially where mass screening is considered.

The high cost of the dip slide is primarily due to the technical difficulty of charging the slide with medium under aseptic conditions, which is very time consuming, whether it is prepared commercially or in our own laboratory.

Most general practitioners instruct the patient how to take midstream specimens, and often tell them to scald out a jar to collect the sample. These instructions may be forgotten or seem unimportant to the patient, and consequently the resulting specimen is often grossly contaminated. Obviously the simpler the method of taking the samples the better, so long as the results give reproducible accuracy.

\section{Method}

This we shall call 'uricount'.

The outfit consists of a polythene screw-capped jar of approximately $2 \mathrm{oz}$ capacity, the diameter of the base being approximately $40 \mathrm{~mm}$. These containers are available from Central Supplies, D.H.S.S. and M.R.C. Central Stores at a cost of $0.6 \mathrm{p}$ each. A blotting paper liner is inserted into the lid and batches are sterilized by ethylene oxide or gamma irradiation. Random samples of these containers, in the condition as received from the supplier, have been tested for bacterial content. About $60 \%$ appeared to be sterile after 24 hours' incubation at $22^{\circ} \mathrm{C}$ and $37^{\circ} \mathrm{C}$; the rest produced a very scanty growth, usually not more than one colony per container.

Into the sterile container is dispensed $5 \mathrm{ml}$ of Received for publication 2 July 1971.

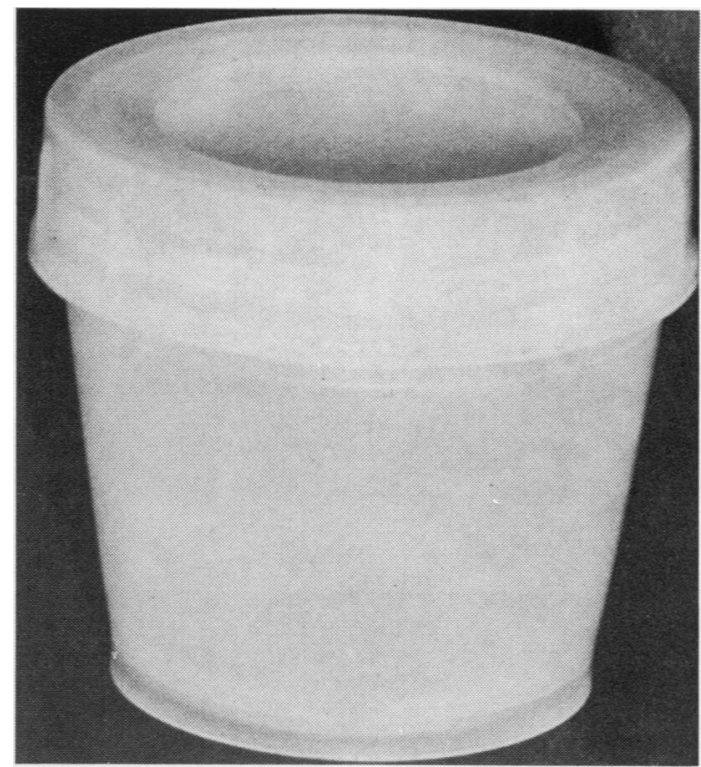

Fig. 1 Uricount container.

melted MacConkey agar to form a layer covering the bottom of the jar, and when this is set the containers are stored either short term (for a few days) at room temperature-or long term in the refrigerator.

If this method is used by general practitioners, where transport by post is involved, it has been found that sometimes, due to gross mishandling by the post office, the medium has slipped in the container. This makes it difficult and occasionally impossible to read the result.

This problem has been overcome by sticking a square or disc of Ford's blotting paper into the bottom of the container with clear Bostick adhesive before sterilization. We recommend also that the concentration of agar in the MacConkey medium be doubled. Tests have been carried out to show that the colony size and the colony count are not affected by these measures.

A leaflet is issued to the patient instructing him how to collect a midstream specimen directly into the container. After allowing the urine to remain in the container for 30 seconds, the contents are poured away or into a sterile Universal container for microscopical and chemical examination. The container is then allowed to drain by holding it upside down for 30 seconds over the lavatory pan. The cap is then replaced and the specimen sent to the laboratory.

Tests were carried out using suspensions of bacteria commonly responsible for urinary tract 


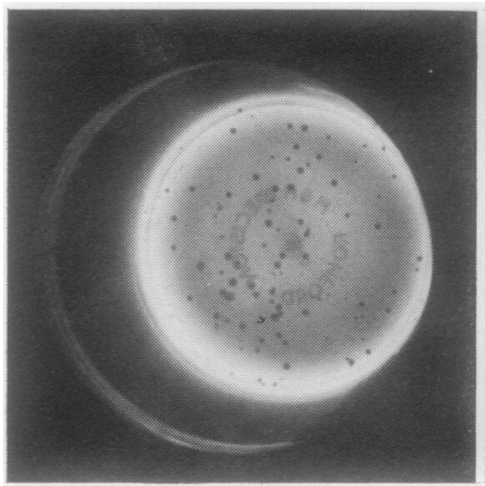

E. coli 1,000 per ml

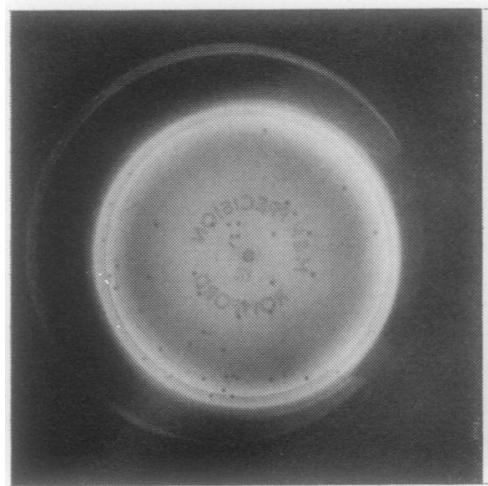

Streptococcus faecalis per $1,000 \mathrm{ml}$

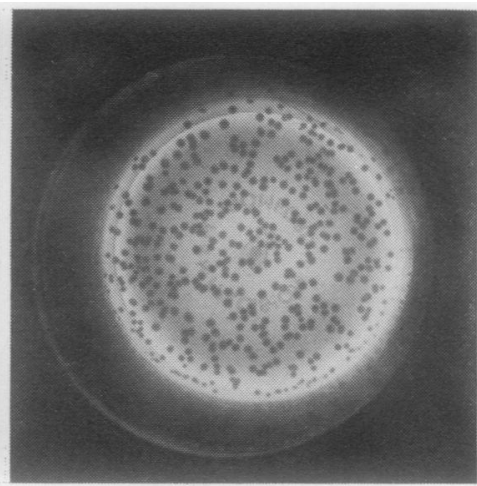

E. coli 10,000 per 10,030 per $\mathrm{ml}$

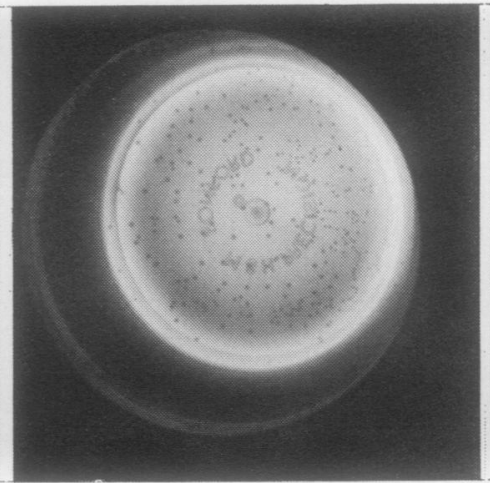

Streptococcus faecalis 10,000 per ml

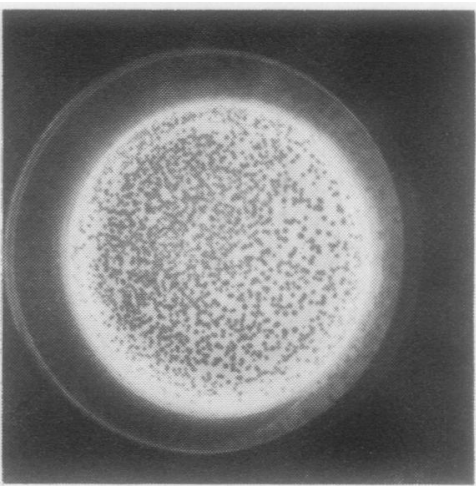

E. coli 100,000 per $\mathrm{ml}$

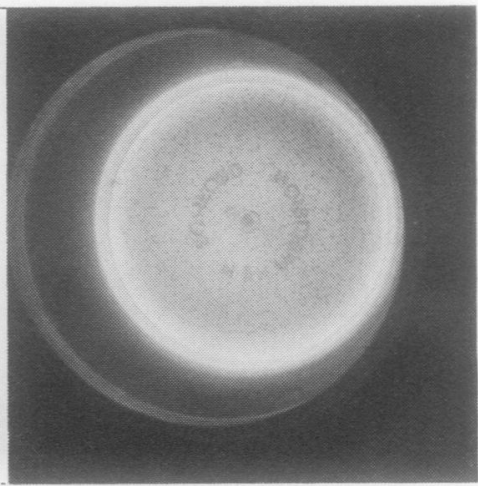

Streptococcus faecalis 100,000 per ml

Fig. 2 Number of colonies obtained from pure cultures.

infections, namely, E. coli, Proteus, coliform bacilli, Streptococcus faecalis, and Staphylococcus aureus.

Ten-fold dilutions of a six-hour broth culture of these organisms in sterile Ringer's solution were prepared from $10^{-1}$ to $10^{-6}$. These suspensions were used for viable counts by the following methods: (1) Miles and Misra viable count (modified); (2) Oxoid dip slide; (3) Uricult (Orion Laboratories; and (4) uricount. They were incubated at $37^{\circ} \mathrm{C}$ overnight. Counts were carried out with the aid of a plate counter.

Results showed that suspensions of organisms containing $10^{5}$ or greater produced an uncountable number of colonies or confluent growth.

Suspensions producing $10^{4}$ to $10^{5}$ colonies by Miles and Misra count produced 25 to 250 colonies on the uricount.

The area of the surface of the medium of the uricount is approximately the same as a Uricult dip slide and consequently a direct comparison can be made. Results by all methods agreed.
The photographs show the number of colonies obtained from bacterial suspensions in pure culture of E. coli and Streptococcus faecalis of 1,000, 10,000 , and 100,000 organisms $/ \mathrm{ml}$.

The time that the urine is actually left in the container was tested at half-minute intervals from 0 to 5 minutes. The resulting number of colonies was not significantly different.

The amount of urine in the container, either just sufficient to cover the surface of the medium or a full container poured off and drained, did not affect the number of colonies significantly.

Tests were carried out to determine whether the medium would contract and allow it to slip out of the container. During all our tests and after storage for two months in the refrigerator the medium layer did not slip.

The uricount has been used routinely for screening for bacteriuria in two antenatal clinics for the past two months. Checks were made by carrying out Miles and Misra counts in duplicate with the uri- 
count on specimens from the clinic in this hospital when the time between collecting and testing the specimen could be controlled. In all cases comparable counts were obtained by both methods.

The second antenatal clinic used in the series is situated several miles from the laboratory and delays in transport of the specimens are inevitable. Results showed the value of the uricount method as many of the specimens examined by conventional methods were grossly overgrown.

\section{Advantages of this Method}

A patient can micturate directly into the container avoiding the use of another sterile container.

The container is cheap, unbreakable, light weight for transport, and is simple to prepare for use by pouring $5 \mathrm{ml}$ of medium into it.
There is very little chance of the patients contaminating the medium with the fingers, as with the slide methods.

After overnight incubation at $37^{\circ} \mathrm{C}$ or at room temperature, the counts can be read quickly and with ease.

The commercial dip slide costs $7 \frac{1}{2} \mathrm{p}$, the uricount container $0.6 \mathrm{p}$, medium $0.2 \mathrm{p}$, plus labour costs.

We wish to thank Dr E. H. Gillespie for his advice and encouragement in the preparation of this paper.

\section{References}

Mackey, J. P., and Sandys, G. H. (1965). Laboratory diagnosis of infections of the urinary tract in general practice by means of a dip-inoculum transport medium. Brit. med. J., 2, 1286-1288.

Naylor, G. R. E., and Guttman, D. (1967). The dip-slide: a modified dip-inoculum transport medium for the laboratory diagnosis of infections of the urinary tract. J. Hyg. (Lond.), 65, 367-371. 